\title{
Elastic Deformation Induced Non-equilibrium Segregation of $P$ in IN718 Alloy
}

\author{
Jinhui Du, Xudong Lu*, Zhongnan Bi, Tingdong Xu \\ Beijing Key Laboratory for Advanced High Temperature Materials, China Iron \& Steel Research Institute Group, Beijing, China
}

Email address:

1xdong0700@hotmail.com (Xudong Lu)

${ }^{*}$ Corresponding author

\section{To cite this article:}

Jinhui Du, Xudong Lu, Zhongnan Bi, Tingdong Xu. Elastic Deformation Induced Non-equilibrium Segregation of P in IN718 Alloy. International Journal of Materials Science and Applications. Vol. 5, No. 2, 2016, pp. 49-53. doi: 10.11648/j.ijmsa.20160502.13

Received: January 25, 2016; Accepted: March 16, 2016; Published: March 25, 2016

\begin{abstract}
IN718 alloy is a precipitation hardened, nickel-based superalloy that is used extensively in the aircraft engine industry. The excellent cyclic fatigue resistance, high tensile strength, fracture toughness and oxidation resistance allow the alloy to be safely used at maximum useful service temperature of about $650^{\circ} \mathrm{C}$. Many researchers improve the alloy's high-temperature strength through adding of P. However, segregation of $\mathrm{P}$ at grain boundary can be induced the grain boundary embrittlement, decreased the tension reduction in area. In this paper, intermediate-temperature embrittlement (ITE) for a IN718 alloy has been experimentally studied by elevated-temperature tension tests. International standard ISO 6892-2 (Metallic Materials Tensile Testing) indicates that the variations in temperature of this measurement system have been found to have a larger potential effect on test results, which is a kind of uncertainty contribution not related to test equipment. International standard ISO $6892-2$ maintains that strain rate variations can induce the measurement uncertainty of mechanical properties in tensile testing, which will imperil the reliability of tension tests. The measurement uncertainty of reduction in area relative to strain rate, called as strain rate embrittlement (SRE), is first described experimentally for IN718 alloy in this paper. Then the measurement uncertainty is clarified based on microscopic theory of elastic deformation in metals. It is shown that the elastic deformation of tension test induced the segregation of impurities to grain boundaries and the relevant embrittlement which produces the measurement uncertainty of reduction in area.
\end{abstract}

Keywords: Elastic Deformation, Non-equilibrium Segregation, IN718 Alloy

\section{Introduction}

IN718 alloy is a precipitation hardened, nickel-based superalloy that is used extensively in the aircraft engine industry, for example, rotor components, such as compressor disks and spools, turbine disks, compressor blades and power drive-shafts, are typical applications. The excellent cyclic fatigue resistance, high tensile strength, fracture toughness and oxidation resistance allow the alloy to be safely used at maximum useful service temperature of about $650^{\circ} \mathrm{C}[1,2,3]$. The precipitation within the fcc $\gamma$-matrix of the coherent, disc-shaped, bct $\left(\mathrm{DO}_{22}\right.$ type superlattice) ordered $\gamma^{\prime \prime}$ precipitate $\left(\mathrm{Ni}_{3} \mathrm{Nb}\right)$ having an average diameter and thickness of about 30 and $10 \mathrm{~nm}$, respectively, is responsible for the contributions of coherency strain hardening and order strengthening. The incoherent equilibrium orthorhombic
$\left(\mathrm{Ni}_{3} \mathrm{Nb}\right)$ with $\delta$-phase precipitate is stable up to about $1010^{\circ} \mathrm{C}$, which plays an important role during deformation processing for improved cyclic fatigue resistance $[4,5]$.

In order to improve the alloy's high-temperature strength, some researchers have focused on enhancing the thermal stability of strengthening phases [6, 7], whereas other researchers obtained good results through micro-alloying. The experimental results of W.D. Cao and R.L. Kennedy indicate, that he stress-rupture life of fine-grained IN718 was increased by $250 \%$ when the P-level was increased to $0.022 \%$ in comparison to the conventional alloys with $0.004 \%$ to $0.009 \%$ [8]. J.H. Du [9] and W.R. Sun [10] demonstrate, that the beneficial role of $\mathrm{P}$ improving stress-rupture life of IN718-type alloys. Segregation of $\mathrm{P}$ at grain boundary lead to the dislocation motion being impeded through P-dislocation interactions and from a reduction of intergranular cracking through increased grain-boundary cohesion. However, 
segregation of $\mathrm{P}$ at grain boundary can be induced the grain boundary embrittlement, decreased the tension reduction in area.

In present paper, the elastic deformation in tension test will be analyzed based on the variation of solute concentration at grain boundaries during anelastic relaxation process. A strain rate embrittlement (SRE) will be suggested, which was induced by the elastic deformation in tension tests. A lot of experimental observations on the strain rate dependence of ductility in variaous alloy systems will be analyzed to indicate the anelastic relaxation inducing indeed a strain rate dependency of embrittlement at grain boundaries in elastic deformation of tension tests. That is the SRE.

The strain rate variations in tension tests can induce the measurement uncertainty of mechanical properties which will imperil the reliability of tension tests [11]. International Standard ISO 6892-2 shows that for a metal, which underwent a same thermal cycle the variations of strain rate in tensile test system can produce change of stress-strain curves which indicates a large difference in testing results, named as measurement uncertainty of mechanical properties relative to strain rate variations in tension test system [11]. It was demonstrated $[12,13,14]$ with the micro-theory of elastic deformation of metals that the stress will change the micro-structure and mechanical properties during the stage of elastic deformation in tension test to induce the measurement uncertainty of mechanical properties. In this paper, the measurement uncertainty of reduction in area relative to strain rate, called as strain rate embrittlement (SRE), is first described experimentally for IN718 alloy. Then the measurement uncertainty is clarified based on microscopic theory of elastic deformation in metals.

In this paper, effect of $\mathrm{P}$ segregation on the tension properties was investigated and micro-mechanism of $\mathrm{P}$ segregation was discussed. Results showed that the $\mathrm{P}$ segregation at grain-boundary is main factor of intermediate-temperature embrittlement (ITE) and strain rate embrittlement (SRE) in IN718 alloy. It is shown that the elastic deformation of tension test induces the non-equilibrium segregation of $\mathrm{P}$ to grain boundaries and the relevant embrittlement which produces the measurement uncertainty of reduction in area in tensile testing.

\section{Experimental Procedures}

The investigations were conducted on $18 \mathrm{~mm}$ diameter hot-roll rods of a commercial IN718 alloy. The chemical composition of this alloy is Ni 53.44, Cr 18.56, Mo 3.02, Nb 5.0, Al 0.44, Ti 1.04, C 0.026, P 0.005, S 0.001, B 0.002, Fe bal (wt.\%). All specimens were first solution at $1050^{\circ} \mathrm{C}$ for $1 \mathrm{~h}$ and then water quenched. Subsequently, the material was aged at $720^{\circ} \mathrm{C} / 8 \mathrm{~h} /$ furnace cooling $(\mathrm{FC})$ to $620^{\circ} \mathrm{C}$ and holding at $620^{\circ} \mathrm{C} / 8 \mathrm{~h} /$ air cooling (AC) to room temperature. The average grain size of all specimens was about $50 \mu \mathrm{m}$. The elevated-temperature tension tests were performed in air at $20-800^{\circ} \mathrm{C}$ using an INSTRON-5582 machine. The reduced cross-section of the test samples was $5 \mathrm{~mm}$ diameter and the gage length was $25 \mathrm{~mm}$. The strain rate was maintained at 2 $\mathrm{mm} / \mathrm{min}$. The holding time at the test temperature prior to the start of tension was $20 \mathrm{~min}$. In this tension test, the reduction in area (RA) was employed as a measure of the ductility of IN718 alloy. At least three identical samples were tested at each tension temperature. The strain rate controlled tension tests were performed at $600^{\circ} \mathrm{C}$ with different strain rate of $0.0001,0.0005,0.001,0.005$ and $0.01 / \mathrm{s}$. At least three identical samples were tested at each tension rate. To identify the relative content of $\mathrm{P}$ at grain boundary, electro probe micro analysis (EPMA) was carried out on selected samples. Samples for EPMA studies were sectioned perpendicular to the loading axis from the gauge section of the failed tension specimens. The samples were mechanically polished and further electrolysis polished.

\section{Results}

Fig. 1 displays the results of elevated-temperature tension tests for the studied alloy and shows a maximum embrittlement at about $650^{\circ} \mathrm{C}$. Maximum embrittlement in the elevated temperature tension tests is induced by a maximum in the grain-boundary concentration of $\mathrm{P}$ at $650^{\circ} \mathrm{C}$ for IN718 alloy. This is due to the fact that the critical time of non-equilibrium grain-boundary segregation (NGS) for $\mathrm{P}$ in this alloy at $650^{\circ} \mathrm{C}$ is closet to $20 \mathrm{~min}$ of holding prior to the start of tension than at other test temperatures [15]. When the test temperatures are lower (higher) than $650^{\circ} \mathrm{C}$, the critical time of $\mathrm{P}$ is longer (shorter) than 20 min with decreasing (increasing) test temperature. So, the embrittlement of this alloy, which is induced by the grain-boundary segregation of $\mathrm{P}$, will get lower and lower with both decreasing and increasing test temperature. Therefore, the intermediate-temperature embrittlement (ITE) of this alloy is produced by the NGS of P.

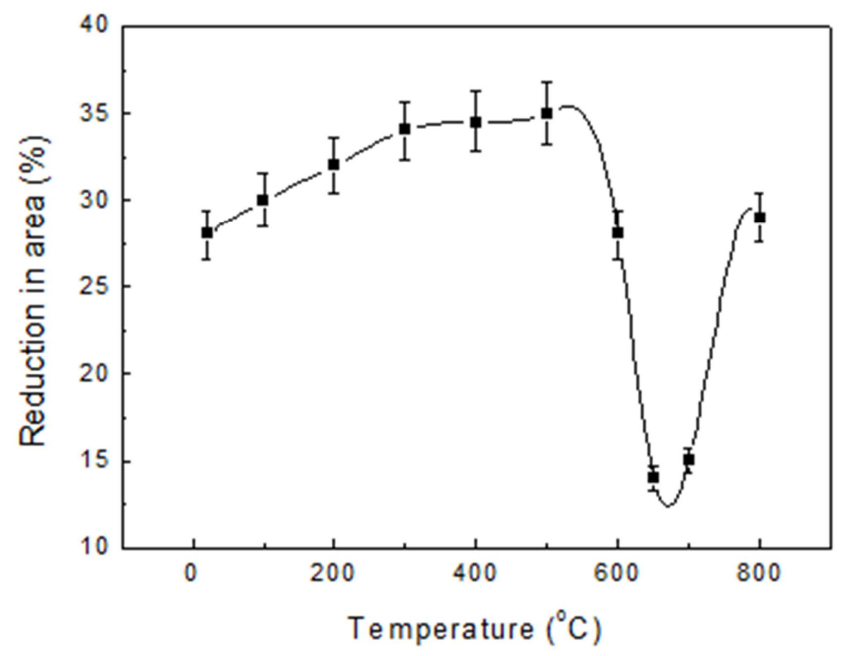

Fig. 1. The reduction in area (RA \%) plotted as a function of tension temperature for IN718 alloy.

Fig. 2 displays the strain rate controlled tension tests results of $600^{\circ} \mathrm{C}$ for IN718 alloy and shows a maximum embrittlement at strain rate $0.001 / \mathrm{s}$. This is due to the fact that 
the critical time for $\mathrm{P}$ in this alloy at $600^{\circ} \mathrm{C}$ is closet to $20 \mathrm{~min}$ of holding prior to the start of tension. For the tension test of IN718 alloy, elastic deformation before plastic deformation is a constant, correspond to the critical time of this temperature, critical strain rate has a determined. The concentration of solute at grain boundaries during the elastic deformation depends on how close the elastic deformation time (EDT) is to the critical time. When EDT is shorter than the critical time, the EDT will get away from the critical time and the segregation concentration decreases with shortening EDT. When EDT is longer than the critical time, the EDT will get close to the critical time and the segregation concentration increases with shortening EDT.

Fig. 3 displays the results of electro probe micro analysis (EPMA) for IN718 alloy and shows $\mathrm{P}$ segregation at grain-boundary. K. Wang [15] was measured the grain boundary concentration for sulfur in the $\mathrm{Ni}-\mathrm{Cr}-\mathrm{Fe}$ alloy by auger electron spectroscopy (AES), results showed that the maximum of the grain boundary concentration for sulfur corresponds to the maximum of embrittlement in the $\mathrm{Ni}-\mathrm{Cr}-\mathrm{Fe}$ alloy at $500^{\circ} \mathrm{C}$. Further work has been quantitatively determine the grain-boundary concentration for $\mathrm{P}$ by AES or three dimensional atom probe (3DAP) in IN718 alloy and find relationship of concentration for $\mathrm{P}$ and grain-boundary embrittlenment.

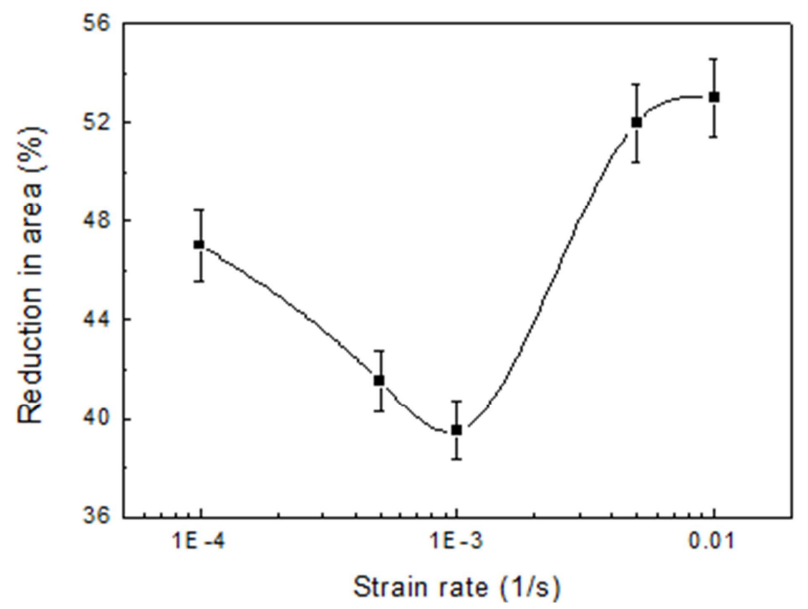

Fig. 2. The reduction in area (RA \%) plotted as a function of tension strain rate at $600^{\circ} \mathrm{C}$ for $I N 718$ alloy.

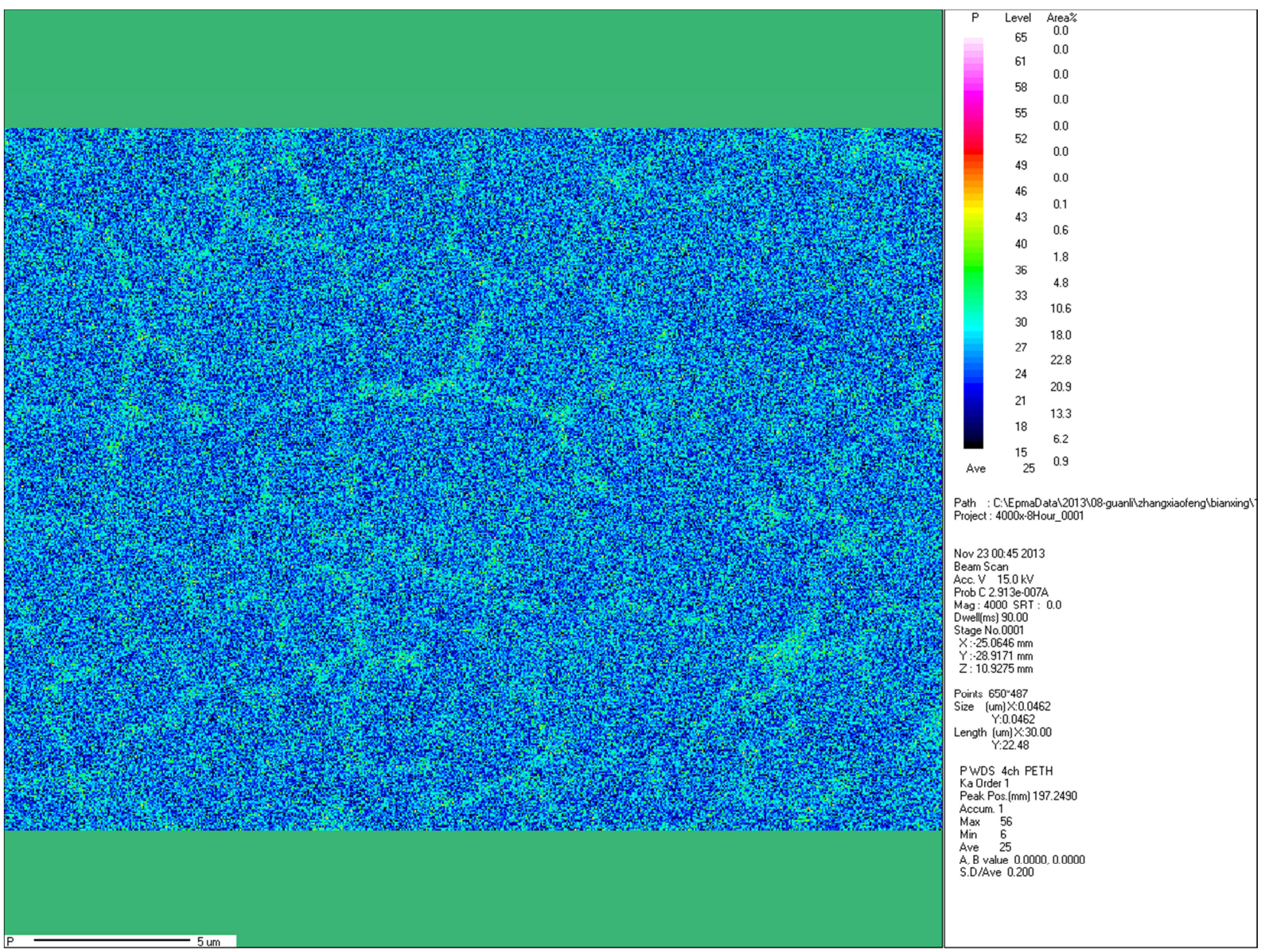

Fig. 3. EPMA image showing the P segregation in grain boundaries.

\section{Discussions}

The non-equilibrium grain boundary segregation (NGS) arising from thermal effects was first reported by Aust et al.
[16], Anthony [17], and Hamneman and Anthony [18]. Faulkner [19] suggested the concept of a critical time. Subsequently, $\mathrm{Xu}$ [20] and Cheng [21] experimentally indicated that the critical time should correspond to the peak in the solute grain-boundary concentration during isothermal 
aging after quenching. Fig. 1 of reference [21] concentration depends on how the aging time approaches the critical time. When the aging time is closer to the critical time, the NGS concentration is high even if the material is over- or under-aged compared to the critical time. This is the characteristic aspect of NGS [21].

The formula for critical time was worked out by Faulkner [19] and Xu et al. [20, 21, 22, 23]:

$$
t_{c}=\frac{r^{2} \ln \left(D_{c} / D_{i}\right)}{\delta\left(D_{c}-D_{i}\right)},
$$

Where $D_{i}$ and $D_{c}$ are the diffusion coefficients for solute atoms and complexes, respectively, $r$ is the grain radius and $\delta$ is the critical time constant. It has been calculated from formula that the critical time will increase monotonically and rapidly with a decrease in aging temperature. This is also a characteristic aspect of the critical time, which has been confirmed many times in $[19,24,25]$.

The variations in Fig. 1 show that the grain boundary concentration of $\mathrm{P}$ reaches a peak after about 20 min during at $650^{\circ} \mathrm{C}$. This means that the critical time of P NGS is about $20 \mathrm{~min}$ at $650^{\circ} \mathrm{C}$ in IN718 alloy.

For the HSLA steels that underwent same thermal cycle and then were tensile tested at a constant temperature with different strain rates, it is shown from Figures 1 to 3 that reduction in area will vary with strain rates. That is strain rate embrittlement. There is usually a strain rate at which reduction in area reaches a minimum for each testing temperature. Such an embrittlement is the measurement uncertainty of reduction in area relative to strain rates. As a result, the steel that should have had one original mechanical property has many different mechanical properties with the variation of strain rate of tension testing. The mechanical property of the measured metal is already changed during the process of tension testing.

An anelastic deformation will preferentially occur at grain boundaries for the elastic deformation of a polycrystalline metal under tension stress at a temperature. Such a micro-mechanism of anelastic deformation was suggested by $\mathrm{Xu}[12,22]$ that vacancies in matrix will be absorbed into grain boundaries which work as a trap of vacancies under a tension stress. Moreover, the T 4 migrating vacancies could drag solute atoms, in a vacancy-solute complex, to grain boundaries to produce an excess concentration of solute there. At the same time, a concomitant but reverse diffusion of solute atoms away from boundary takes place along the solute concentration gradient established. Accordingly, a stress ageing time must exist at which the solute diffusion balances the complex diffusion and the solute concentration at grain boundary reaches a maximum value.

When subjected to a uniaxial elastic stress, a polycrystalline metal increases length in the direction of the applied stress and the elastic deformation occurs during which the metal atoms are displaced from their original positions but not to the extent that they take up new positions. Metal may exhibit a time-dependent and recoverable deformation, known as anelastic deformation in addition to the purely elastic, time-independent deformation. Based on the observations above, such a micro-mechanism of anelastic deformation in metals was suggested that vacancies in matrix will be absorbed into grain boundaries which work as a trap of vacancies under a tension stress. Moreover, the migrating vacancies could drag solute atoms, in a vacancy-solute complex, to the grain boundaries to produce an excess concentration of solute at grain boundaries. At the same time, a concomitant but reverse diffusion of solute atoms away from boundary takes place along the solute concentration gradient established. Accordingly, a stress ageing time must exist at which the solute diffusion balances the complex diffusion and the solute concentration at grain boundary reaches a maximum value. This stress ageing time is called the critical time of elastic deformation. The absorption of vacancies into grain boundaries will stop when the stress equilibrium under a tensile stress is reached during anelastic relaxation of elastic deformation. In such case, the concentrations of vacancy and solute at grain boundaries were formulated, called as equilibrium equations. The kinetic equations were also formulated for grain boundary segregation of elastic deformation in metals, which present analytically the variation of solute concentration at grain boundaries with the stress ageing time (or elastic deformation time) under an applied tensile stress.

The grain boundary embrittlement induced by elastic deformation of tension tests have been identified on the following two aspects: a) for the tension tests with a constant strain rate at variant temperatures, there must be such a test temperature the critical time of which is equal or close to the EDT of the strain rate. The grain boundary embrittlement will reach maximum at the test temperature. Such a test temperature dependency of grain boundary embrittlement is called as the intermediate temperature embrittlement, which occurs ubiquitously in metals and alloys. b) For the tension tests at a constant temperature with various stain rates the EDT will increase with decreasing the strain rate at test temperature and the segregation will also changed [12]. Hence a strain rate dependency of embrittlement occurs at grain boundaries. There is a strain rate at which the EDT of the tension test will be equal or close to the critical time of the test temperature and the SRE will reach maximum.

The procedure of tension tests includes an elastic deformation stage and a plastic deformation stage to fracture. Whether the plastic deformation of tension test will play role on the SRE or not? It was pointed out by Chen and Song [26] that the plastic deformation can enhance the segregation of impurity to grain boundaries in interstitial free steel. At a certain strain rate the impurity boundary concentration increases with increasing plastic deformation until reaching a steady value. That means that there is no a critical time and the relative concentration peak at grain boundaries during the plastic deformation of tension tests. As a result the plastic deformation will not be responsible for the SRE and its CSR (critical strain rate) of the tension tests. Therefore, the elastic deformation of tension test served to initiate the intergranular segregation and cavities, and plastic deformation to fracture 
served to complete the fracture along the weakened grain boundaries.

\section{Conclusions}

In this paper, intermediate-temperature embrittlement (ITE) for a IN718 alloy has been experimentally studied by elevated-temperature tension tests. The measurement uncertainty of reduction in area relative to strain rate, is first described experimentally for IN718 alloy. Then the measurement uncertainty is clarified based on microscopic theory of elastic deformation. It is shown that the elastic deformation of tension test induced the segregation of impurities to grain boundaries and the relevant embrittlement which produces the measurement uncertainty of reduction in area. Further work has been quantitatively determine the grain-boundary concentration for $\mathrm{P}$ by AES or three dimensional atom probe (3DAP) in IN718 alloy and find relationship of concentration for $\mathrm{P}$ and grain-boundary embrittlenment. Conclusions as follow:

(1) The intermediate-temperature embrittlement and strain rate embrittlement exist in the tension tests for IN718 alloy. The elastic deformation in tensile test can induce the no-equilibrium segregation of $\mathrm{P}$ at grain boundaries to embrittlement the grain boundaries.

(2) A maximum of strain rate embrittlement in the curves of RA-strain rate for IN718 alloy occurs at a critical strain rate at a certain test temperature. The critical strain rate will always move to a slower strain rate with decrease in test temperatures.

\section{References}

[1] J. M. Zhang, Z. Y. Gao, J. Y. Zhuang, Z. Y. Zhong, P. Janschek, J. Mater. Proc. Tech. 70 (1997) p. 252.

[2] C. M. Kuo, Y. T. Yang, H. Y. Bor, C. N. Wei, C. C. Tai, Mater. Sci. Eng. A, 510-511 (2009) p. 289.

[3] S. Coste, E. Andrieu, J. Huez, Mater. Sci. Eng. A, 396 (2005) p. 92.

[4] J. Warren, D. Y. Wei, Mater. Sci. Eng. A, 428 (2006) p. 106.

[5] S. Azadian, L. Y. Wei, R. Warren, Mater. Character. 53 (2004) p. 7.
[6] J. M. Oblak, D. F. Paulonis, D. S. Duvall, Metall. Trans. 5A (1974) p. 143.

[7] J. P. Collier, S. H. Wong, J. C. Phillips, J. K. Tien, Metall. Trans. 19A (1988) p. 1657.

[8] W. D. Cao, R. L. Kennedy, Superalloy 1996, TMS, (1996) p. 589.

[9] X. D. Lu, J. H. Du, Q. Deng, Mater. Sci. Eng. A, 559 (2013) p. 623.

[10] W. R. Sun, S. R. Guo, D. Z. Lu, Z. Q. Hu, Metall. Mater. Trans. A28 (1997) p. 649.

[11] International Standard, ISO 6892-2, Metallic Materials-Tensile Tsting-Part 2: Method of Test at Elevated Temperature, First Edition 2011-02-15.

[12] T. D. Xu, L. Zheng, K. Wang, R. D. K. Misra, Inter. Mater. Rev. 58(5) (2013) p. 263.

[13] T. D. Xu, C. Shao, K. Wang, Acta Phys. Sin. 63 (2014) p. 1.

[14] T. D. Xu, Z. W. Zheng, Z. J. Liu, H. Y. Yu, Measurement, 66 (2015) p. 1.

[15] K. Wang, T. D. Xu, Y. Q. Wang, J. H. Du, Philo. Mag. Lett. 89 (2009) p. 725.

[16] K. T. Aust, R. E. Hanneman, P. Niessen and J. H. Westbrook, Acta Metall. 16 (1968) p. 291.

[17] T. R. Anthony, Acta Metall. 17 (1969) p. 603.

[18] R. E. Hamneman and T. R. Anthony, Acta Metall. 17 (1969) P. 1133.

[19] R. G. Faulkner, J. Mater. Sci. 16 (1981) p. 373.

[20] T. D. Xu, J. Mater. Sci. Lett. 7 (988) p. 241.

[21] T. D. Xu and B. Y. Cheng, Prog. Mater. Sci. 49 (2004) p. 109.

[22] T. D. Xu, J. Mater. Sci. 22 (1987) p. 337.

[23] T. D. Xu and S. H. Song, Acta Metall. 37 (1989) p. 2499.

[24] T. D. Xu, Phil. Mag. Lett. 86(2006) p. 501.

[25] X. L. He, Y. Y. Chu and J. J. Jonas, Acta Metall. 37 (11) p. 2905.

[26] X. M. Chen, S. H. Song, L. Q. Weng, K. Wang. Mater. Sci. Eng. A 545 (2012) p. 86. 